\title{
THE RESEARCH OF INDUCED CURRENT IN COILS WHEN THE PROCESS OF MOTION OF MAGNETICALLY LEVITATED PLANAR ACTUATORS
}

\author{
Rougang Zhou ${ }^{1, \mathrm{a}}$, Yunfei Zhou ${ }^{1, \mathrm{~b}}$, Guangdou Liu ${ }^{1, \mathrm{c}}, \mathrm{Xiao} \mathrm{Tu}^{1, \mathrm{~d}}$ \\ ${ }^{1}$ State Key Lab of Digital Manufacturing Equipment \& Technology, Huazhong \\ University of Science \& Technology, Wuhan, P.R.,430074,China ; \\ aemail:rougangzhou@hust.edu.com, bemail:_yfzhou@mail.hust.edu.cn, \\ cemail: lgd-5401@163.com, demail:_1006336923@qq.com
}

\begin{abstract}
In the process of motion magnetically levitated planar actuators, the magnetic flux through the coils been changed as the position changed, It produced inductive electromotive force which associated with the location and speed of coils. Induction electromotive force to produce induced current in the coils, which influence the stability of the actuators. This paper presents a analytical model of induction electromotive force in the coils of magnetically levitated a planar actuators, According to the analytical model it could predict control the compensation of drive currents in order to offset the induced current which produce by electromotive force, the stability of Magnetically levitated planar actuators have been improved. Finite element simulation had been used in this paper to approve the correct of the analytical model.
\end{abstract}

\section{KEYWORDS}

Induced Current, Magnetically Levitated Planar Actuators, Halbach Permanent Magnet array

\section{INTRODUCTION}

Magnetically levitated planar actuators can realize high-accuracy and wear-free high-speed and friction-less motion[1-6]. So, they have become the focus of the research on the high-accuracy and high-speed long-stroke motion system. Because the motion part of magnetically levitated planar actuator is six-degree-of-freedom levitation motion, a minor disturbance could cause the instability and kinematic accuracy. The drive current accuracy of coils is the one of critical point of control precision and stability of magnetically levitated planar actuators. The papers [2] have indicated that the magnetic flux in the coils have been changed which according to the speed and position of the actuators, hence it produce induction electromotive force. Induced current have been produced by induction electromotive force which influence the stability of the drive current accuracy. In order to solve this question the predict control offset should be adopted. The recent researches have not performed the analytical model of induced current in magnetically levitated planar actuators. For this reason, this paper theoretically analyzes the induced current regularity in the motion of magnetically levitated planar actuators. Also, the paper proposes the analytical model for the induced current by the coils when the motion of the actuators. The comparison of the analytical model results with the finite element calculation shows that the analytical model in 
this paper yields correct results and can further provide a induced current compensation method for the precise magnetic levitation motion control.

\section{The Characteristic of Permanent Magnet Planar ARRay}

The topology structure of Magnetically levitated planar actuators with moving coils as shown in figure(1)[1-3], It is composed of Halbach permanent magnet array and coils which suspended on it, the coils are arranged in an angle of $45^{\circ}$ with the Halbach magnet array in order to product the force applied to coils in the $\mathrm{x}$ and $\mathrm{y}$ direction is physically decoupling. The global coordinate system is defined at the stationary part of the Halbach permanent magnet array [2,3]:

$$
\vec{x}=\left[\begin{array}{lll}
x & y & z
\end{array}\right]^{T}
$$

A local coordinate system is defined in the volume center point of the coil, it is denoted with the superscript ${ }^{c}$

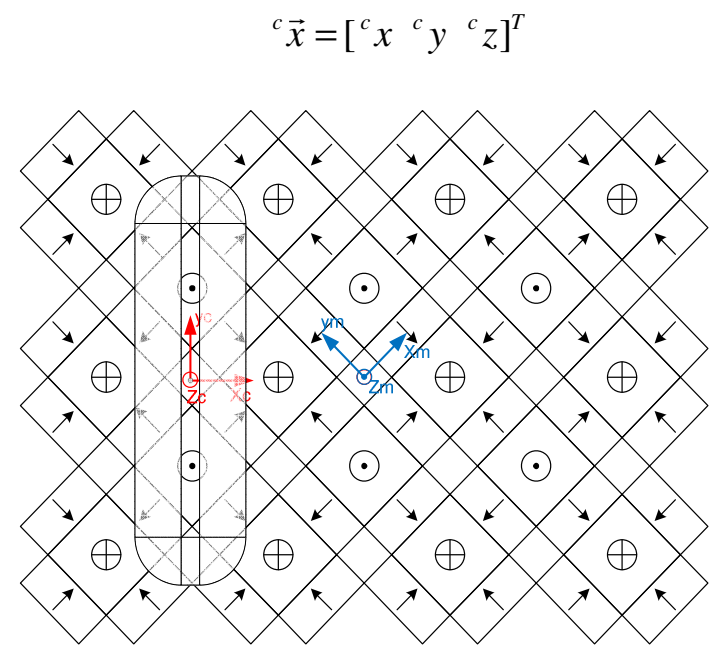

(a)

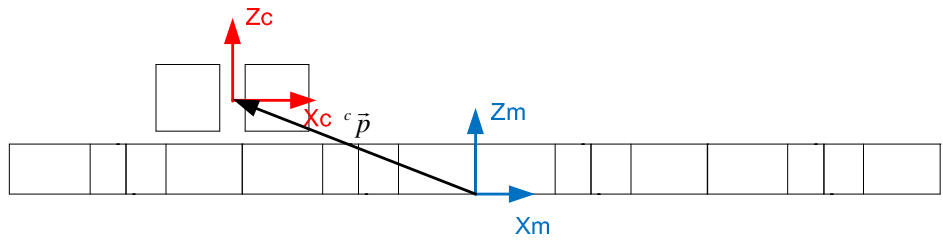

(b)

Fig. 2 Bottom and side view of the components of a magnetically levitated stage with moving coils a)cross section b) Bottom view.

The magnetic flux density expression in local coordinate system is simplified to[2]: 


$$
\overrightarrow{{ }^{c} B}=\left[\begin{array}{l}
-\frac{B_{x y}}{\sqrt{2}} e^{-\frac{\sqrt{2} \pi}{\tau} c^{c} z} \sin \left(\frac{{ }^{c} x \sqrt{2} \pi}{\tau}\right) \\
\frac{B_{x y}}{\sqrt{2}} e^{-\frac{\sqrt{2} \pi}{\tau}{ }^{c} z} \sin \left(\frac{{ }^{c} y \sqrt{2} \pi}{\tau}\right) \\
\frac{1}{2} B_{z} e^{-\frac{\sqrt{2} \pi}{\tau} c^{c}}\left(\cos \left(\frac{{ }^{c} x \sqrt{2} \pi}{\tau}\right)-\cos \left(\frac{{ }^{c} y \sqrt{2} \pi}{\tau}\right)\right)
\end{array}\right]
$$

The $B_{z}$ component of the magnetic flux density is the factor that produced induction electromotive force in the coils, as shown in Fig. (3).

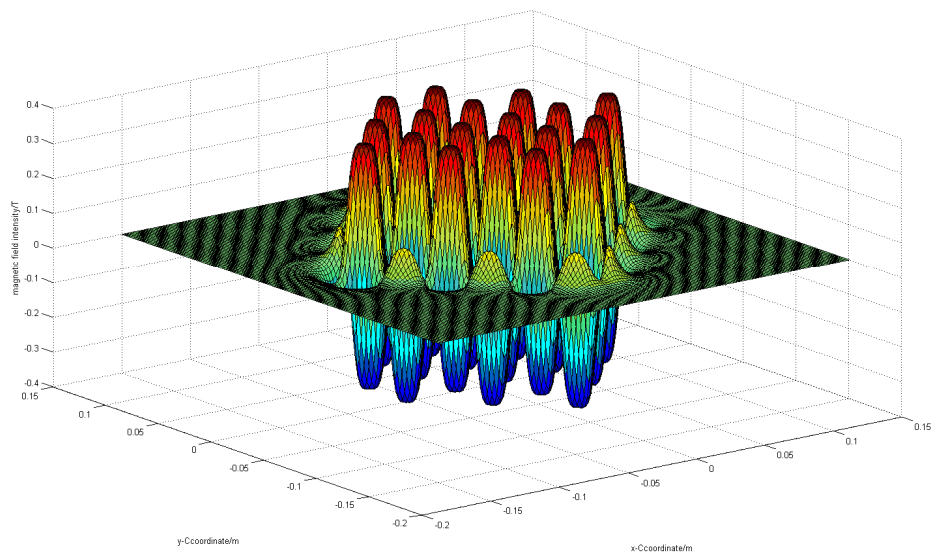

Figure 3: component in the $\mathrm{Z}$ direction of the magnetic field

\section{THE ANALYZE OF INDUCED CURRENT IN MOTION COILS}

According the paper[2,3], in the filaments model, current could been seen as through the center of the coils. And it could be adopted in the analyzed of induced current in motion coils. As show in figure(1), defining 4 lines in the center of the coils, and the $\mathrm{Z}$ coordinate of the lines is[23]:

$$
{ }^{c} r_{z}=c_{t}-{ }^{c} p_{z}-\frac{1}{\lambda}-\frac{\left(c_{b}-c_{t}\right) e^{\lambda c_{b}}}{e^{\lambda c_{b}}-e^{\lambda c t}}
$$

Because the motion range of coils in Z-direction is small (less than $1 \mathrm{~mm}$ ), it is not care the Induction electromotive force which has been produced by motion of coils in Z-direction, if the coils have horizontal motion. According the formula of inductive electromotive force:

$$
\xi_{a b}=\int_{a}^{b}(v \times B) \cdot d l
$$

The induction electromotive force of the coils when moving in $\mathrm{x}, \mathrm{y}$ direction could obtain. Combine the size and structure of the Halbach permanent magnet array it could deduce that the induction electromotive force be produced when the coils have y- velocity component, the zdirection of magnetic field produce induction electromotive force in 1,3 sides of the coils which can be simply added,2,4 sides don't produce induction electromotive force. When the coils have $\mathrm{y}$ - velocity component, 1,3 sides don't produce induction electromotive force and the z-direction of magnetic field produce induction electromotive force in 1,3 sides of the coils which is offset. So the induction electromotive force of the moving coils is: 


$$
\xi_{c o i l}=\int_{a 1}^{b 1}\left(v_{y} \times B_{z}\right) \cdot d l_{1}+\int_{a 2}^{b 2}\left(v_{y} \times B_{z}\right) \cdot d l_{2}
$$

And the induced current of the moving coils is:

$$
I=\frac{\boldsymbol{\xi}_{\text {coil }}}{R_{\text {coil }}}
$$

From Formula[BZ], it can be deduced that induced current is:

$$
I=v_{y} \frac{-2 \cos \left(\frac{{ }^{c} y \sqrt{2} \pi}{\tau}\right) \sqrt{2} \tau B_{z} e^{-\frac{\sqrt{2} \pi}{\tau} c z}}{R_{\text {coil }}}
$$

\section{VERTIFICATION RESULTS AND DISCUSSION}

The model structure is as shown in Fig. (1). For a coil and a Halbach magnet array with dimension as shown in Table (1),

Dimensions of coil and Halbach magnet array during calculation

\begin{tabular}{|c|c|c|}
\hline Parameter & Value & Unit \\
\hline Pole pitch magnet array, $\tau_{l}$ & 21 & $\mathrm{~mm}$ \\
\hline Pole pitch magnet array, $\tau_{n}$ & 10 & $\mathrm{~mm}$ \\
\hline Pole pitch of magnetic cycle, $\tau$ & $\tau=\tau_{l}+\tau_{n}$ & $\mathrm{~mm}$ \\
\hline Coil length,$l$ & 81.4 & $\mathrm{~mm}$ \\
\hline $\begin{array}{c}\text { Remanence of permanent } \\
\text { magnets }, B\end{array}$ & 1.24 & $T$ \\
\hline magnet height,$m h$ & 7.0 & $\mathrm{~mm}$ \\
\hline coil height, $h$ & 9.2 & $\mathrm{~mm}$ \\
\hline Coil width, $c w$ & 22.8 & $\mathrm{~mm}$ \\
\hline velocity y-direction,$x$ & 2 & $\mathrm{~m} / \mathrm{s}$ \\
\hline $\begin{array}{c}\text { Mechanical clearance }(z- \\
\text { direction), } m c\end{array}$ & 1 & $\mathrm{~mm}$ \\
\hline The thickness of the layers,$b$ & 7.23 & $\mathrm{~mm}$ \\
\hline
\end{tabular}

The induced current that the coil is subject to when it moves along the $\mathrm{x}$ axis at a speed of $2 \mathrm{~m} / \mathrm{s}$ is as shown in Fig.[], it can be seen that the results from the analytical model are consistent with the periodic rules of the simulation results and the amplitudes are identical. 


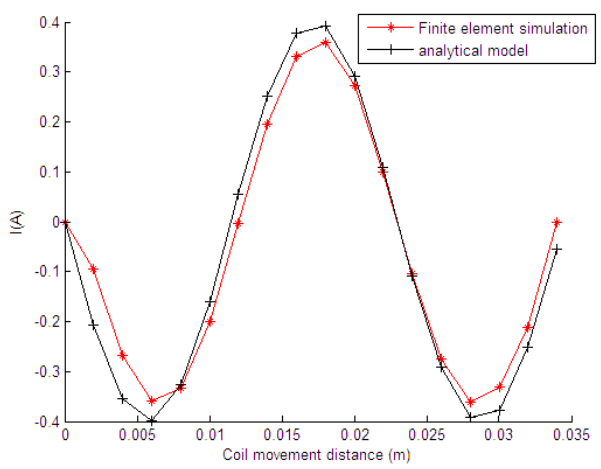

Fig. Comparison of induced current of the coil when the coil moves at $1 \mathrm{~m} / \mathrm{s}$ in $y$-direction.

\section{CONCLUSIONS}

In the magnetically levitated planar actuator, the induced current are generated in the moving foilwound coils and it can't be ignored which exert influence over the stability of the Magnetically levitated planar actuator. This paper analyzes and calculates the induced current generated in the coils when the coils move above the Halbach magnet array. The induced current analytical model has been given and finite element simulation deduced in this paper. According compare the results, it is demonstrates that the analytical model is correct. it is possible to conduct the compensation calculation in the precise motion control.

\section{REFERENCES}

[1] Jansen, J. W., Van Lierop, C. M. M., Lomonova, E. A., \& Vandenput, A. J. A. (2007). Modeling of magnetically levitated planar actuators with moving magnets. Magnetics, IEEE Transactions on, 43(1), 15-25.

[2] Jansen, J. W. (2007). Magnetically levitated planar actuator with moving magnets: Electromechanical analysis and design. Einhoven: Technische Universiteit Einhoven.

[3] Rovers, J. M. M., Jansen, J. W., Compter, J. C., \& Lomonova, E. A. (2012). Analysis method of the dynamic force and torque distribution in the magnet array of a commutated magnetically levitated planar actuator. Industrial Electronics, IEEE Transactions on, 59(5), 2157-2166.

[4] Rovers J M M, Stöck M, Jansen J W, et al. (2013)Real-time 3D thermal modeling of a magnetically levitated planar actuator. Mechatronics,240 - 246.

[5] Peng, Junrong, and Yunfei Zhou. (2013)"Modeling and Analysis of a New 2-D Halbach Array for Magnetically Levitated Planar Motor. IEEE Transactions on,: 1-1.

[6] Rovers J M M, Jansen J W, Compter J C, et al. (2012), Analysis method of the dynamic force and torque distribution in the magnet array of a commutated magnetically levitated planar actuator. Industrial Electronics, IEEE Transactions on, 59(5), 2157-2166.

\section{AUTHORS}

Rougang Zhou was born in Zhejing,China.. Now ,he is a PhD Candidate of State Key Lab of Digital Manufacturing Equipment \& Technology, Huazhong University of Science. 